\title{
Observer Based Controller For Single Track Vehicles
}

\author{
Habib Dabladji, Dalil Ichalal, Hichem Arioui and Saïd Mammar
}

\begin{abstract}
The present work deals with the observer basedcontroller design for nonlinear Powered Two-Wheelers Vehicles (PTW) modeled in Takagi-Sugeno fuzzy representation. Based on the Lyapunov method and Linear Matrix Inequality (LMI) formulation, sufficient conditions have been derived to prove the Input to State Stability (ISS) property of the nonlinear PTW model with such a controller.

The main contribution of this paper is that the whole stability of the couple observer-controller is studied by designing the observer and the controller separately and proving the ISS of the whole nonlinear PTW model. The aim here is to ensure a best profile tracking of the PTW in term of roll preview (trajectory) for large range of longitudinal velocities.
\end{abstract}

\section{INTRODUCTION}

In the last decade, the car fleet increased significantly with the motorcycles one. Several works were carried out in order to enhance safety and comfort to automobile conductors. However, a significant time delay can be clearly noted in the case of motorcycles. This can be confirmed by the increase of motorcycle's fatalities (20 times higher when driving a car) [1].

The time delay in the development of control safety systems for motorcycles can be explained by different factors. Economically, the PTW market is less interesting comparing to car one and the resources in research and program development are paltry. Moreover, riders believe that they do not need safety and restrictive systems which alter their experience or their freedom of riding. Finally, from an engineering point of view, PTW are inherently unstable nonlinear systems whose properties strongly depend on travel velocity.

To this fact, the design of stabilizing or safety controller for such vehicles is particularly challenging. To our knowledge, the study proposed in [5] were probably the first to stabilize a motorcycle using nonlinear equations of motion derived from a highly simplified model. Since the $21^{\text {st }}$ century, many contributions have been given to the modeling and the study of motorcycles dynamics [2], [11], [17]. Nevertheless, a few works have been done on the control of motorcycles and a little more on the observation and the estimation of state variables and external inputs. In [13], a robust feedback stabilization based on a linear model at low speed has been presented. Optimal control techniques have been explored in [9] and [18]. In the second one, it has been combined with preview control and gain scheduling techniques. But in both works, any proof of the global stability of the vehicle in all riding conditions has

All the authors are with the IBISC Laboratory, Evry-Val-d'Essonne University, 40, rue de Pelvoux, 91020 Evry Courcouronne Cedex habib.Dabladji@ibisc.univ-evry.fr been given. In [15], an optimal control has been suggested based on the projection operator approach, but the controllers are optimized for a specific maneuver. In [4], [7] Genetic algorithms and fuzzy techniques have been used to control motorcycles for a set of forward velocities and roll angles, but as like as said before, we cannot get any proof of the stability in all riding conditions. Based on a 2 degrees of freedom model, a linearizing control for trajectory tracking for an autonomous motorcycle was proposed in [22].

Furthermore, sparsely works deal with the estimation of the dynamics of PTW. The estimate of the roll angle was proposed in [19] but with the neglect of the steering dynamics. The observation of steer angle was proposed in [3] with a scheduling gains missing the estimation convergence guarantee. More recently, a higher order sliding mode observer was proposed to estimate the lateral dynamics and steering torque in [12]. The same authors proposed also a study on state and unknown input estimation by exploring the Takagi-Sugeno systems and linear matrix inequalities in [6].

To the best of our knowledge, the simultaneous estimation and control of the lateral dynamics of PTW with a study of the closed-loop response have never been addressed for a large range of longitudinal velocity.

The paper is organized as follows: in section II, we state the problem. In section III, we present the motorcycle model used in this work. The observer and the controller are developed in section IV and V. The simulation results of this work are given in section VI. We finish by the conclusion and the appendix.

\section{Problem statement}

The main objective of this work is to design a TS-fuzzy observer based controller in order to estimate the important unmeasurable state variables that affect the lateral dynamics of PTW (roll angle $(\phi)$, lateral velocity $\left(v_{y}\right)$ and the lateral forces $\left(F_{y f}\right.$ and $\left.F_{y r}\right)$ ), to guarantee the whole stability of the PTW and at the same time to ensure a trajectory tracking objective which is given by a reference roll angle (a safe roll preview with respect to a velocity).

The class of systems that will be treated is given by equations (1).

$$
\left\{\begin{array}{l}
\dot{x}(t)=\sum_{i=1}^{n} \mu_{i}(x) A_{i} x(t)+B_{1} r(t)+B_{2} u(t) \\
h_{1}(t)=C_{11} x(t) \\
h_{2}(t)=C_{12} x(t) \\
y(t)=C_{2} x(t)+D w(t)
\end{array}\right.
$$

$x(t) \in \mathbb{R}^{n \times 1}$ is the state vector, $u(t) \in \mathbb{R}^{n_{u} \times 1}$ is the control input, $y(t) \in \mathbb{R}^{n_{y} \times 1}$ is the output vector, $r(t) \in \mathbb{R}^{1 \times 1}$ and 
$w(t) \in \mathbb{R}^{n_{w} \times 1}$ are respectively the reference signal and the noise measurement and $h_{1}(t) \in \mathbb{R}^{n_{h 1} \times 1}$ and $h_{2}(t) \in \mathbb{R}^{n_{h_{2}} \times 1}$ are respectively the sensitivity signals of the observer and the controller. Note that the premise variables depend on unmeasurable state variables.

Because of the presence of uncontrollable external signals (noise measurements and reference signal) and unmeasurables premise variables, we cannot apply the separation principle to prove the stability of the observer-based controller. To this end, we use the property of Input to State Stability (ISS) that will guarantee the boundedness of the state estimation error and trajectory tracking error.

In what follow and to prove the convergence of the observer based-controller proposed in this work, the following definition is used.

Definition 1: [8] Given a system $\Sigma$ and its state trajectory defined by: $\dot{x}(t)=f(x(t), u(t))$. The system $(\Sigma)$ verifies the Input To State Stability (ISS) if there exists a $\mathscr{K} \mathscr{L}$ function $\beta: \mathbb{R}^{n} \times \mathbb{R} \longrightarrow \mathbb{R}$ and a $\mathscr{K}$ function $\alpha: \mathbb{R} \longrightarrow \mathbb{R}$ such that for each input $u(t)$ satisfying $\|u(t)\|_{\infty}<\infty$ and each initial conditions $x(0)$, the trajectory of $(\Sigma)$ associated to $x(0)$ and $u(t)$ satisfies:

$$
\|x(t)\|_{2} \leq \beta(\|x(0)\|, t)+\alpha\left(\|u(t)\|_{\infty}\right)
$$

The definition of a $\mathscr{K} \mathscr{L}$ and a $\mathscr{K}$ function can be found in the same refernce.

\section{MOTORCYCLE DYNAMICS}

\section{A. Nonlinear model}

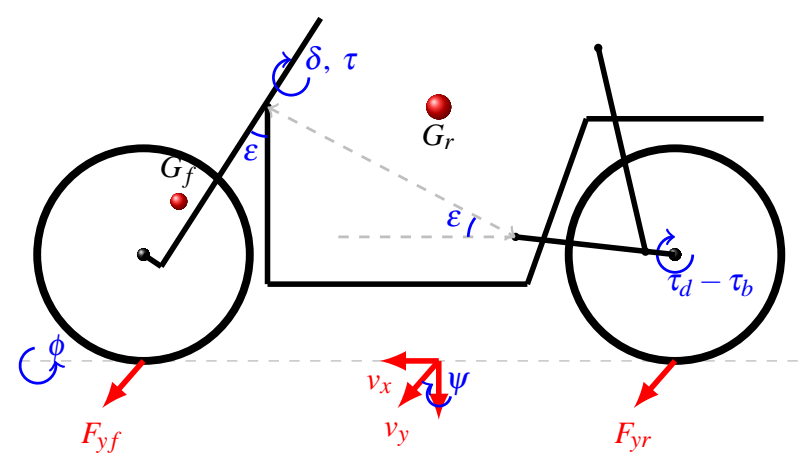

Fig. 1. Geometrical representation of the Sharp's motorcycle model

Here, the lateral dynamics of a motorcycle are represented by a model with four equations [16] due essentially to the effect of lateral forces from the front and rear wheels $\left(F_{y f}\right.$ and $F_{y r}$ ) and the yaw and roll motions under rider's steering actions. The study of such a model aims to reconstruct the state variables of a motorcycle in cornering situation.

These motions are expressed by the following equations which correspond respectively to the lateral, yaw, roll and steering dynamics:

$$
\left\{\begin{array}{l}
M \dot{v}_{y}+M_{f} k \ddot{\psi}+\left(M_{f} j+M_{r} h\right) \ddot{\phi}+M_{f} e \ddot{\delta}=F_{y f}+F_{y r} \\
M_{f} k\left(\dot{v}_{y}+v_{x} \dot{\psi}\right)+a_{2} \ddot{\phi}+a_{3} \ddot{\psi}+a_{1} \ddot{\delta}-a_{4} v_{x} \dot{\phi} \\
-\frac{i_{f y}}{R_{f}} \sin \varepsilon v_{x} \dot{\delta}+M_{f} k v_{x} \dot{\psi}=\sum M_{z} \\
\left(M_{f} j+M_{r} h\right) \dot{v}_{y}+b_{2} \ddot{\phi}+a_{2} \ddot{\psi}+b_{1} \ddot{\delta}+b_{5} v_{x} \dot{\psi} \\
+\frac{i_{f y}}{R_{f}} \cos \varepsilon v_{x} \dot{\delta}=\sum M_{x} \\
M_{f} e \dot{v}_{y}+b_{1} \ddot{\phi}+a_{1} \ddot{\psi}+c_{1} \ddot{\delta}-\frac{i_{f y}}{R_{f}} \cos \varepsilon \dot{\phi}+c_{3} v_{x} \dot{\psi} \\
+K \dot{\delta}=\sum M_{s}
\end{array}\right.
$$

where:

$$
\left\{\begin{array}{l}
\sum M_{z}=l_{f} F_{y f}-l_{r} F_{y r} \\
\sum M_{x}=b_{4} \sin (\phi)-b_{3} \sin (\delta) \\
\sum M_{s}=-b_{3} \sin (\phi)-c_{2} \sin (\delta)-\eta F_{y f}+\tau
\end{array}\right.
$$

The lateral forces $F_{y f}$ and $F_{y r}$ acting, respectively, on the front and rear wheels depend on the sideslip angles $\alpha_{f}$ and $\alpha_{r}$ and the camber angles $\gamma_{f}$ and $\gamma_{r}$, and are expressed by:

$$
\left\{\begin{array}{l}
\frac{\sigma_{f}}{v_{x}} \dot{F}_{y f}=-F_{y f}-C_{f 1} \alpha_{f}+C_{f 2} \gamma_{f} \\
\frac{\sigma_{r}}{v_{x}} \dot{F}_{y r}=-F_{y r}-C_{r 1} \alpha_{r}+C_{r 2} \gamma_{r}
\end{array}\right.
$$

where $\alpha_{f}=\left(\frac{v_{y}+l_{f} \dot{\psi}-\eta \dot{\delta}}{v_{x}}\right)-\delta \cos (\varepsilon), \alpha_{r}=\left(\frac{v_{y}-l_{r} \dot{\psi}}{v_{x}}\right)$, $\gamma_{f}=\phi+\delta \sin (\varepsilon)$ and $\gamma_{r}=\phi$

In this work, a normal riding is considered (without taking into account the limit situations) which justifies the linear form of the lateral forces $F_{y f}$ and $F_{y r}$ with respect to both sideslip and camber angles.

By adding the mathematical expressions of the forces to the motorcyle dynamics and by choosing the state vector as $x(t)=\left[\begin{array}{llllllll}\phi & \delta & v_{y} & \dot{\psi} & \dot{\phi} & \dot{\delta} & F_{y f} & F_{y r}\end{array}\right]^{T}$, the system is rewritten as follows:

$$
E \dot{x}(t)=\tilde{A}\left(x(t), v_{x}\right) x(t)+\tilde{B} \tau(t)
$$

The obtained model is the same as the one used in [6], [10]. We suggest readers to refer to this articles for more details on the matrices $E, \tilde{B}$ and $\tilde{A}(x)$ and their parameters.

\section{B. Exact TS model of the nonlinear motorcycle model and augmented system with sensitivity signals}

In order to express the model in TS fuzzy structure, let us consider the following nonlinearities:

$$
z_{1}=v_{x}, \quad z_{2}=\frac{\sin (\phi)}{\phi}
$$

It can be seen that only two nonlinearities are considered in the model presented in the previous section. This is due to small values taken by the steering angle $\delta(t)$, and the consideration that the lateral forces obey to linear equations. We suppose also that the roll angle does not exceed $45^{\circ}$

Without loss of generality, the motorcycle model can be rewritten as:

$$
\dot{x}(t)=\sum_{i=1}^{4} \mu_{i}\left(v_{x}, x\right) A_{i} x(t)+B \tau(t), y(t)=C x(t)+D w(t)
$$

where $A_{i}=E^{-1} \tilde{A}_{i}, B=E^{-1} \tilde{B}$ and $\tilde{A}_{i}$ corresponds to the TS formulation of $\tilde{A}, y$ is the output vector and $w(t)$ includes measurement noises. In order to ensure a state trajectory tracking with respect to a desired roll angle, we added a 
new state variable given by: $e_{\phi}=\int_{0}^{t}\left(\phi-\phi_{r}\right) d t$, where $\phi$ is the real roll angle and $\phi_{r}$ is the one to be tracked.

We add also sensitivity signals. The signal $h_{1}$ corresponds to the observable part that should be sensitive to noise measurement. We choose our sensitivity signals as high pass filters with a cut-off frequency of $20 \mathrm{~Hz}$ to filter high frequencies of noises measurements, where $e_{i}$ is the state estimation error that corresponds to $\left(x-x_{e}\right)$. The second sensitivity signal $h_{2}$ corresponds to the controller part. It is chosen as a lower pass filter. Its cut-off frequency is chosen equal to the biggest unstable frequency of motorcycles which corresponds to the wobble mode (about $8 \sim 10 \mathrm{~Hz}$ ).

$$
h_{1 i}=\frac{3 s}{s+30 \pi} e_{i}, h_{2}=\frac{40 \pi}{s+20 \pi}\left(\phi-\phi_{r}\right)
$$

Thus, we will obtain an augmented TS-fuzzy system as described in (1). In what follow, we may omit sometimes time variable $t$ for brevity and we write $\mu_{i}$ and $\hat{\mu}_{i}$ instead of $\mu_{i}\left(v_{x}, x\right)$ and $\mu_{i}\left(v_{x}, \hat{x}\right)$ respectively.

\section{OBSERVER DESIGN}

The objective of this section is to design a nonlinear observer for TS-fuzzy systems.

The following nonrestrictive assumptions can be made.

Assumption 1: In the remaining it is supposed that

- The state $x$ is bounded (stable or stabilized motorcycle)

- The pairs $\left(A_{i}, C_{2}\right)$ are observable

- The measurement noise signals are bounded

The first assumption holds in open loop for a reduced longitudinal speed range and the pairs $\left(A_{i}, C_{2}\right)$ are considered to be observable to guarantee solutions to the LMI problem.

\section{A. State estimation}

Let us consider the nonlinear observer given by

$$
\dot{\hat{x}}=\sum_{i=1}^{4} \hat{\mu}_{i}\left(A_{i} \hat{x}+L_{i}(y-\hat{y})\right)+B_{1} r+B_{2} u, \hat{y}=C_{2} \hat{x}
$$

Let us consider the state estimation error $e(t)=x(t)-\hat{x}(t)$. The matrices $L_{i} \in \mathbb{R}^{n \times n_{y}}$ of the observer are to be determined. Using equations (1) and (8), we obtain the following state space representation:

$$
\dot{e}=\sum_{i=1}^{4} \hat{\mu}_{i} \Phi_{i} e+\Delta-L_{i} D w, h_{1}=C_{11} e
$$

where: $\Phi_{i}=A_{i}-L_{i} C_{2}, \Delta=\sum_{i=1}^{4}\left(\mu_{i}-\hat{\mu}_{i}\right) A_{i} x$

According to the hypothesis of assumption 1 and the convexity of $\mu_{i}$, the terms $\Delta$ and $w$ are also bounded.

\section{B. Observer's convergence study}

The observer (8) will be synthesized by solving the optimization problem under LMI constraints given in the Theorem 1 in order to ensure the convergence of the observer.

Theorem 1: Under the Assumption 1, if there exists a symmetric and positive definite matrix $P_{1}$, gain matrices $Z_{i}$ and positive scalars $\varepsilon_{1}, \varepsilon_{2}$ and $\varepsilon_{3}$ solution to the following optimization problem, $i=1, \ldots, 4$

$$
\min _{P_{1}, Z_{i}, \varepsilon_{1}, \varepsilon_{2}, \varepsilon_{3}} \zeta_{1} \varepsilon_{1}+\zeta_{2} \varepsilon_{2}+\zeta_{3} \varepsilon_{3}
$$

s.t.

$$
\left(\begin{array}{cccc}
\Omega_{i} & P_{1} & -Z_{i} D & C_{11}^{T} \\
P_{1} & -\varepsilon_{1} I & 0 & 0 \\
-\left(Z_{i} D\right)^{T} & 0 & -\varepsilon_{1} I & 0 \\
C_{11} & 0 & 0 & -\varepsilon_{3} I
\end{array}\right)<0
$$

where: $\Omega_{i}=A_{i}^{T} P_{1}+P_{1} A_{i}-Z_{i} C_{2}-C_{2}^{T} Z_{i}^{T}$

and $\zeta_{1}, \zeta_{2}$ and $\zeta_{3}$ are positive weighting scalars.

Thus the state errors are bounded. The gains of the observer are computed from $L_{i}=P_{1}^{-1} Z_{i}$. The attenuation level of the transfers from $\Delta(t)$ and from $w(t)$ to state estimation error are bounded and given respectively by the quantities $\sqrt{\frac{\varepsilon_{1} \varepsilon_{3}}{\left\|C_{11}\right\|_{2}^{2}}}$ and $\sqrt{\frac{\varepsilon_{2} \varepsilon_{3}}{\left\|C_{11}\right\|_{2}^{2}}}$.

The state estimation errors guarantee the Input To State Stability (ISS) is given by the following property:

$\|e(t)\|_{2}<\sqrt{\frac{\lambda_{\max }\left(P_{1}\right)}{\lambda_{\min }\left(P_{1}\right)}}\|e(0)\|_{0} e^{-\frac{\eta}{2} t}+\sqrt{\frac{\varepsilon_{1} \varepsilon_{3}}{\left\|C_{11}\right\|_{2}^{2}}}\|\Delta(t)\|_{\infty}+\sqrt{\frac{\varepsilon_{2} \varepsilon_{3}}{\left\|C_{11}\right\|_{2}^{2}}}\|w(t)\|_{\infty}$

The scalars $\varepsilon_{i}$ are then chosen sufficiently small in order to have the minimal transfer from $\Delta(t)$ and $w(t)$ to state estimation error.

Proof: In order to prove the convergence of the state estimation errors, let us consider the following Lyapunov function: $V_{1}(t)=e^{T}(t) P_{1} e(t)$

According to the equation (9), the time derivative of $V_{1}(t)$ is given by:

$$
\begin{aligned}
\dot{V}_{1}(t) & =\sum_{i=1}^{4} \hat{\mu}_{i} e^{T}\left(\Phi_{i}^{T} P_{1}+P_{1} \Phi_{i}\right) e(t) \\
& +e^{T} P_{1} \Delta+\Delta^{T} P_{1} e-e^{T} P_{1} L_{i} D w-w^{T} D L_{i}^{T} P_{1} e
\end{aligned}
$$

To attenuate the transfer from the perturbation signals to the sensitivity signals, we must satisfy (14) with $\left.\varepsilon_{i}\right|_{(i=1, \ldots 3)}$ are positive scalars:

$$
\varepsilon_{3}^{-1} h_{1}^{T} h_{1}<\varepsilon_{1} \Delta^{T} \Delta+\varepsilon_{2} w^{T} w
$$

A sufficient condition to satisfy the stability of the error estimation and the inequality (14) is to solve the following LMI (15):

$$
\dot{V}_{1}(t)+\varepsilon_{3}^{-1} h_{1}^{T} h_{1}-\varepsilon_{1} \Delta^{T} \Delta-\varepsilon_{2} w^{T} w<0
$$

Which is equivalent to (16):

$$
\left(\begin{array}{ccc}
\Omega_{i}+\varepsilon_{3}^{-1} C_{11}^{T} C_{11} & P_{1} & \Gamma_{i} \\
P_{1} & -\varepsilon_{1} I & 0 \\
\Gamma_{i}^{T} & 0 & -\varepsilon_{2} I
\end{array}\right)<0
$$

where $\Gamma_{i}=Z_{i} D$

Applying the Schur's lemma, we can easily find the LMI given in theorem (1).

Knowing that:

$$
\lambda_{\text {min }}\left(P_{1}\right)\|e(t)\|_{2}^{2} \leq V_{1}(t) \leq \lambda_{\text {max }}\left(P_{1}\right)\|e(t)\|_{2}^{2}
$$

one obtains from (15) the inequality:

$$
\dot{V}_{1}<-\eta_{1} V_{1}+\varepsilon_{1} \Delta^{T} \Delta+\varepsilon_{2} w^{T} w
$$

where $\eta_{1}$ is a positive scalar given by: $\eta_{1}=\frac{\varepsilon_{3}^{-1}\left\|C_{11}\right\|_{2}^{2}}{\lambda_{\min }\left(P_{1}\right)}$ 
It follows:

$$
\begin{aligned}
V_{1}(t) & <V_{1}(0) e^{-\eta_{1} t} \\
& +\varepsilon_{1} \int_{0}^{t} e^{-\eta_{1}(t-s)}\|\Delta(s)\|_{2}^{2} d s+\varepsilon_{2} \int_{0}^{t} e^{-\eta_{1}(t-s)}\|w(s)\|_{2}^{2} \\
& <V_{1}(0) e^{-\eta_{1} t}+\frac{\varepsilon_{1}}{\eta_{1}}\|\Delta(t)\|_{\infty}^{2}+\frac{\varepsilon_{2}}{\eta_{1}}\|w(t)\|_{\infty}^{2}
\end{aligned}
$$

Using again the relation (17), we obtain:

$$
\begin{aligned}
\|e(t)\|_{2}^{2} & <\frac{\lambda_{\max }\left(P_{1}\right)}{\lambda_{\min }\left(P_{1}\right)}\|e(0)\|_{2}^{2} e^{-\eta_{1} t} \\
& +\frac{\varepsilon_{1}}{\eta_{1} \lambda_{\min }\left(P_{1}\right)}\|\Delta(t)\|_{\infty}^{2}+\frac{\varepsilon_{2}}{\eta_{1} \lambda_{\min }\left(P_{1}\right)}\|w(t)\|_{\infty}^{2} \\
& <\frac{\lambda_{\max }\left(P_{1}\right)}{\lambda_{\min }\left(P_{1}\right)}\|e(0)\|_{2}^{2} e^{-\eta_{1} t} \\
& +\frac{\varepsilon_{1} \varepsilon_{3}}{\left\|C_{11}\right\|_{2}^{2}}\|\Delta(t)\|_{\infty}^{2}+\frac{\varepsilon_{2} \varepsilon_{3}}{\left\|C_{11}\right\|_{2}^{2}}\|w(t)\|_{\infty}^{2}
\end{aligned}
$$

This is equivalent to (12) by using the square root.

According to Lyapunov formulation of Input To State Stability (ISS), the state errors converge to a region which will be minimized in order to achieve a more accurate estimation of the vehicle states. This region is as small as the constants $\varepsilon_{i}$ are smaller. To enhance the performances of the observer, a minimal values of these quantities are obtained by minimizing the cost function given by (10).

\section{Controller DESign}

The objective of this section is to design a fuzzy controller based on the estimated states.

As like as in the previous section, the following nonrestrictive assumptions can be made.

Assumption 2: In the remaining it is supposed that

- The pairs $\left(A_{i}, B\right)$ are controllable

- The reference signal is bounded

\section{A. Feedback control law}

The controller proposed in this work is based on the state estimation. Its aim is to make the motorcycle stable in all defined forward velocities. Moreover, we try to track a state space trajectory which is given by a reference roll angle.

Considered the system given by (1) and the control law: $u=-\sum_{i=1}^{4} \hat{\mu}_{i} K_{i} \hat{x}$. Thus, the closed loop system is given by:

$$
\dot{x}=\sum_{i=1}^{4} \hat{\mu}_{i} \Xi_{i} x+B_{1} r+\Delta+B_{2} K_{i} e, h_{2}=C_{12} x
$$

where: $\Xi_{i}=A_{i}-B_{2} K_{i}$

\section{B. Closed-loop Observer based controller's convergence study}

In the absence of measurement noise, we can easily apply the separation principle to design an observer and a controller separately (see [21] for more details). But in our case, by designing them separately, we cannot prove the stability of the observer based controller. Knowing that $\Delta$ and $r$ are bounded and as like as in the previous section, we will prove the ISS of the closed-loop system together with the observer part.

Theorem 2: Under the Assumption (2), if there exists a symmetric and positive definite matrix $X_{2}$, gain matrices $Y_{i}$ and positive scalars $\varepsilon_{4}, \varepsilon_{5}$ and $\varepsilon_{6}$ solution to the following optimization problem, $i=1, \ldots, 4$

$$
\min _{X_{2}, Y_{i}, \varepsilon_{4}, \varepsilon_{5}, \varepsilon_{6}} \zeta_{4} \varepsilon_{4}+\zeta_{5} \varepsilon_{5}+\zeta_{6} \varepsilon_{6}
$$

s.t.

$$
\left(\begin{array}{cccc}
\Pi_{i} & I & B_{1} & X_{2} C_{12}^{T} \\
I & -\varepsilon_{4} I & 0 & 0 \\
B_{1}^{T} & 0 & -\varepsilon_{5} I & 0 \\
C_{12} X_{2} & 0 & 0 & -\varepsilon_{6} I
\end{array}\right)<0
$$

where: $\Pi_{i}=A_{i} X_{2}+X_{2} A_{i}-B_{2} Y_{i}-Y_{i}^{T} B_{2}^{T}$

and $\zeta_{4}, \zeta_{5}$ and $\zeta_{6}$ are positive weighting scalars.

Then the state vector is bounded. The gains of the controller are computed from $K_{i}=Y_{i} X_{2}^{-1}$. The attenuation level of the transfers from $\Delta(t)$, from $r(t)$ and from $w(t)$ to state vector and to state estimation error is bounded and depends proportionately to scalars $\varepsilon_{i}(i=1, \ldots, 6)$.

The scalars $\varepsilon_{i}$ are then chosen sufficiently small in order to have the minimal transfer from disturbance signals to state vector.

Proof:

In order to prove the stability of the state vector $x(t)$, let us consider the following Lyapunov function: $V_{2}(t)=x^{T}(t) P_{2} x(t)$

According to the equation (21), the time derivative of $V_{2}(t)$ is given by:

$$
\begin{aligned}
\dot{V}_{2}(t) & =\sum_{i=1}^{4} \hat{\mu}_{i}\left(x^{T} \Upsilon_{i} x\right)+f(e) \\
& +x^{T} P_{2}\left(\Delta+B_{1} r\right)+\left(\Delta^{T}+r^{T} B_{1}^{T}\right) P_{2} x
\end{aligned}
$$

where: $\Upsilon_{i}=P_{2}\left(A_{i}-B_{2} K_{i}\right)+\left(A_{i}-B_{2} K_{i}\right)^{T} P_{2} \quad$ and $f(e)=\sum_{i=1}^{4} \hat{\mu}_{i}\left(x^{T} B_{2} K_{i} e+e^{T} K_{i}^{T} B_{2}^{T} x\right)$

Noting that combining equations (13) and (24), we obtain a BMI problem. A solution to this problem was proposed in [20] by resolving the LMIs in 2 steps but they lead to a lot of conservatism for the observer based-controller solution.

Inspired by [21], we use the separation principle to prove the ISS stability of our system with perturbation and unmeasurable premise variables by treating each part separately and without taking into account the bilinear terms in the solution.

Let us consider: $d V_{2 a}(t)=\dot{V}_{2}-f(e)$

To attenuate the transfer from the perturbation signals to the sensitivity signals, we must satisfy (25):

$$
\varepsilon_{6}^{-1} h_{2}^{T} h_{2}<\varepsilon_{4} \Delta^{T} \Delta+\varepsilon_{5} r^{T} r
$$

We propose to solve the following LMI (26):

$$
d V_{2 a}(t)+\varepsilon_{6}^{-1} h_{2}^{T} h_{2}-\varepsilon_{4} \Delta^{T} \Delta-\varepsilon_{5} r^{T} r<0
$$

This yields to the following inequality:

$$
\left(\begin{array}{ccc}
\Upsilon_{i}+\varepsilon_{6}^{-1} C_{12}^{T} C_{12} & P_{2} & P_{2} B_{1} \\
P_{2} & -\varepsilon_{4} I & 0 \\
B_{1}^{T} P_{2} & 0 & -\varepsilon_{5} I
\end{array}\right)<0
$$


Note that the LMI (27) is equivalent to (23) given in theorem (2) by multiplying at left and at right by a Matrix $T\left(T=\operatorname{diag}\left[P_{2}^{-1} I I\right]\right)$ and applying the Schur's lemma with $X_{2}=P_{2}^{-1}$ and $Y_{i}=K_{i} X_{2}$. If the later holds, this implies:

$$
\dot{V}_{2}(t)<-\eta_{2}\|x\|_{2}^{2}+\tilde{w}^{T} \tilde{\varepsilon} \tilde{w}+f(e)
$$

where: $\eta_{2}=\varepsilon_{6}^{-1}\left\|C_{12}\right\|_{2}^{2}$ and $\tilde{w}=\left(\Delta^{T} r^{T}\right)^{T}$

Using the fact that:

$$
\begin{aligned}
\lambda_{\min }\left(P_{2}\right)\|x\|_{2}^{2} & \leq V_{2}(t) \\
\frac{\partial V_{2}}{\partial x} & \leq \eta_{3}\|x\|_{2} \\
\eta_{3} & >0
\end{aligned}
$$

This imply:

$$
\begin{aligned}
f(e) & =\frac{\partial V_{2}^{T}}{\partial x} \sum_{i=1}^{4} \hat{\mu}_{i} B_{2} K_{i} e \\
& <\eta_{3} \sum_{i=1}^{4} \hat{\mu}\left\|B_{2} K_{i}\right\|_{2}\|x\|_{2}\|e\|_{2}
\end{aligned}
$$

one obtains the inequality:

$$
\dot{V}_{2}<-\eta_{2}\|x\|_{2}^{2}+\alpha\|x\|_{2}\|e\|_{2}+\tilde{w}^{T} \tilde{\varepsilon} \tilde{w}
$$

with $\alpha>\sum_{i=1}^{4} \hat{\mu}\left\|B_{2} K_{i}\right\|_{2}$

It follows

$$
\begin{aligned}
\dot{V}_{2} & <-\frac{\eta_{2}}{2}\|x\|_{2}^{2} \\
& -\frac{\eta_{2}}{2}\left(\|x\|_{2}^{2}-\frac{2 \alpha}{\eta_{2}}\|x\|_{2}\|e\|_{2}+\left(\frac{\alpha}{\eta_{2}}\|e\|_{2}^{2}\right)-\left(\frac{\alpha}{\eta_{2}}\|e\|_{2}^{2}\right)\right) \\
& +\tilde{w}^{T} \tilde{\varepsilon} \tilde{w} \\
& <-\frac{\eta_{2}}{2}\|x\|_{2}^{2}+\frac{\alpha}{2 \eta_{2}}\|e\|_{2}^{2}+\tilde{w}^{T} \tilde{\varepsilon} \tilde{w} \\
& <-\frac{\eta_{2}}{2 \lambda_{\max }\left(X_{2}\right)} V_{2}+\frac{\alpha}{2 \eta_{2} \lambda_{\max }\left(P_{1}\right)} V_{1}+\tilde{w}^{T} \tilde{\varepsilon} \tilde{w}
\end{aligned}
$$

Combining (18) with (35), we obtain:

$$
\begin{aligned}
\left(\begin{array}{l}
\dot{V}_{1} \\
\dot{V}_{2}
\end{array}\right) & <\left(\begin{array}{cc}
-\eta_{1} & 0 \\
\frac{\alpha}{2 \eta_{2} \lambda_{\max }\left(P_{1}\right)} & -\frac{\eta_{2}}{2 \lambda_{\max }\left(X_{2}\right)}
\end{array}\right)\left(\begin{array}{l}
V_{1} \\
V_{2}
\end{array}\right) \\
& +\left(\begin{array}{ccc}
\varepsilon_{2} & 0 & \varepsilon_{3} \\
\varepsilon_{4} & \varepsilon_{5} & 0
\end{array}\right)\left(\begin{array}{c}
\|\Delta\|_{2}^{2} \\
\|r\|_{2}^{2} \\
\|w\|_{2}^{2}
\end{array}\right)
\end{aligned}
$$

The matrix multiplied by the vector $\left(V_{i}\right)$ is a negative definite matrix. As explained in the proof of theorem 1, this confirms the ISS of the observer based controller and the convergence of the all the state variables to a region which will be minimized in order to achieve a more accurate estimation of the states, stability of the controlled system and an accurate trajectory tracking. This ball is as small as the constants $\varepsilon_{i}$ are smaller. To enhance the performances of the observer, a minimal values of these quantities are obtained by minimizing the cost function given by (10) and (22).

\section{Simulation RESUlts}

The nonlinear system, including longitudinal and lateral dynamics of two-wheeled vehicle is used. It requires three inputs: the rider's steering torque applied on the handlebars (which is our control input) and the two longitudinal torques applied on both front and rear wheels. It takes into account all the important nonlinearities of motorcycle model and moreover the lateral forces are used in their nonlinear form given in [14]. The control of the longitudinal part has not been taken in consideration.

The observer estimating the lateral dynamics and steering torque using only the measured states $\dot{\psi}$ and $\dot{\phi}$ given by the inertial unit and $\dot{\delta}$ and $\delta$ obtained from a suitable encoder. The longitudinal velocity is considered varying from $10 \mathrm{~m} / \mathrm{s}$ to $20 \mathrm{~m} / \mathrm{s}$

In the absence of noise measurement the results are in figures $(2,3)$. We see that all the state variables are well estimated except the lateral velocity, but the boundedness of the error estimation is always guaranteed.
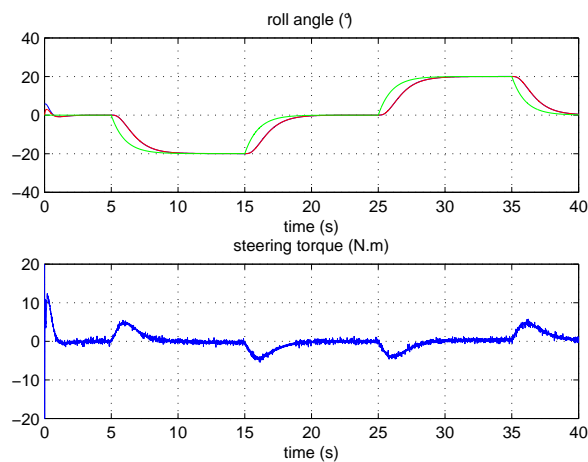

Fig. 2. (top) actual roll angle (blue) and estimated roll angle (below) and reference roll angle (green) (right) steering torque
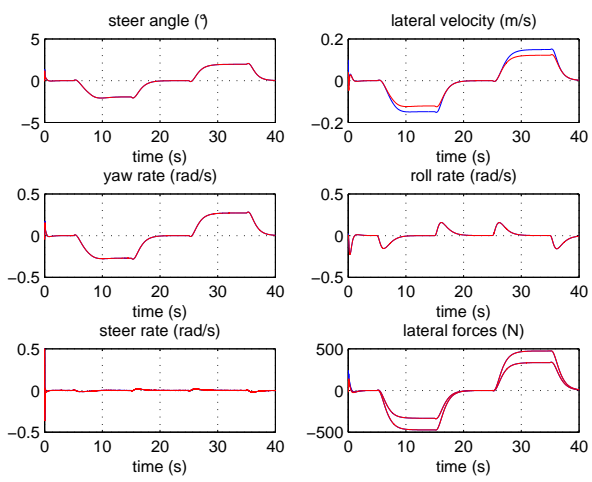

Fig. 3. actual states (blue) and estimated states (red)

In order to test the observer in the presence of measurement noise, let us consider the same observers parameters and assume that the measurement signals are affected by a centered and random noise with magnitude $5 \%$ of the maximal values of the measured variables. The obtained results are depicted in the figures $(4,5)$. The states are then 
well estimated except also the lateral velocity but it is always bounded.
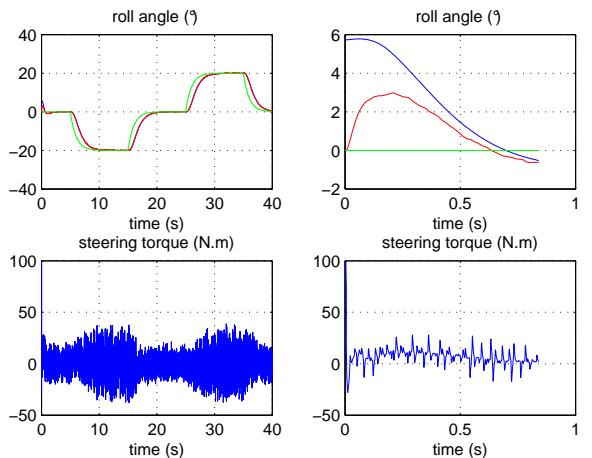

Fig. 4. (top) roll angle and a zoom on it (blue) actual roll angle (red) estimated roll angle (green) reference roll angle (below) steering torque and a zoom on it
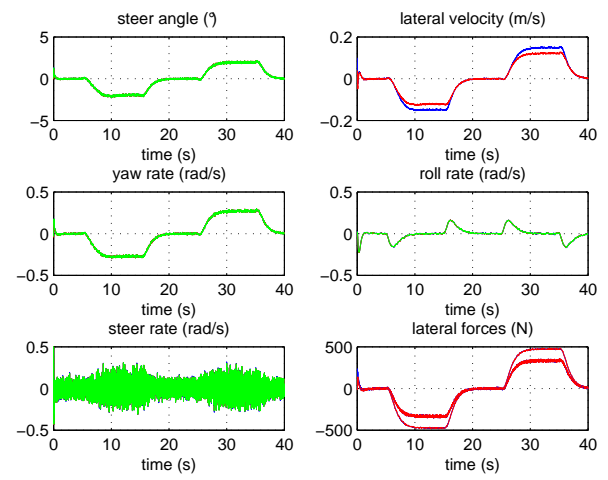

Fig. 5. actual states (blue) and estimated states (red) and measured states (green)

We see that in both cases, only the lateral velocity has significant error estimation. The lateral velocity is one of the hardest states to observe accurately and this can be confirmed by the well-conditioned nature of the observability and controllability matrices. However; because of the insignificant values of the lateral velocity compared to the longitudinal one, we can explain why this error does not affect the performances of the roll angle tracking.

\section{CONCLUSION}

In this paper, an observer based controller is proposed to estimate the lateral dynamics of an autonomous single track vehicle and to track a desired roll angle. A nonlinear model is then considered and transformed to a TS fuzzy structure. The observer based controller is designed to ensure a best profile tracking of the PTW in term of roll preview for a large range of longitudinal velocities. The specificity of this work is the inclusion of the noise measurement and the unmeasurable premise variables. Moreover and from the engineering point of view, to our knowledge, designing a controller or an observer based controller and proving its stability for a large range of forward velocities has never been done.
The convergence study of the couple observer-controller is studied using Lyapunov theory and LMI conditions are given to ease the design of the observer based controller. It is pointed out that this couple guarantees the ISS property. Simulation results are provided to illustrate the effectiveness of the proposed observer based controller even with noise measurements. In future work, the nonlinear model will be redefined to take into account the dynamics of an actuation system. This will be achieved in order to automate a scooter and to get a steering torque more filtered. Some validation results with this motor will be published in future works.

\section{REFERENCES}

[1] Characteristics and causes of road accidents, 2011. Technical report, French Interministerial National Observatory of Road Safety, 2012.

[2] V. Cossalter and R. Lot. A motorcycle multi-body model for real time simulations based on the natural coordinates approach. Vehicle System Dynamics, 37:423 - 447, 2002.

[3] P. De Filippi, M. Corno, M. Tanelli, and S.M. Savaresi. Singlesensor control strategies for semi-active steering damper control in two-wheeled vehicles. Vehicular Technology, IEEE Transactions on, 61(2):813-820, 2012.

[4] S. Fujii, S. A. Panfilov, and S. V. Ulyanov. A model for motorcycle rider operation based on genetic algorithms. Yamaha Mot Tech Rev, 38:132 - 139, 2004.

[5] N. Getz. Dynamic Inversion of Nonlinear Maps with Applications to Nonlinear Control and Robotics. PhD thesis, Department of Electrical Engineering and Computer Sciences, University of California, 1995.

[6] D. Ichalal, H. Dabladji, H. Arioui, and S. Mammar. Observer design for motorcycle lean and steering dynamics estimation: a takagi-sugeno approach. In American Control Conference (ACC), 2013.

[7] G. Inoue and S. Yasunobu. An intelligent MIMO control for twowheeled vehicle. In Fuzzy Systems Conference, 2007.

[8] H.K. Khalil. Nonlinear systems. Macmillan Pub. Co., 1992.

[9] M. Massaro, V. Cossalter, and R. Lot. A virtual rider for reproducing experimental manoeuvres. In Bicycle and Motorcycle Dynamics, 2010.

[10] L. Nehaoua, H. Arioui, and S. Mammar. Motorcycle riding simulator: How to estimate robustly the rider's action. Vehicular Technology, IEEE Transactions on, 62(1):80-88, 2013.

[11] L. Nehaoua, H. Arioui, N. Seguy, and S. Mammar. Dynamic modeling of a two wheeled vehicle : Jourdain formalism. Vehicle System Dynamics, 51(5):648 - 670, February 2013.

[12] L. Nehaoua, D. Ichalal, H. Arioui, S. Mammar, and L. Fridman. Lean and steering motorcycle dynamics reconstruction: An unknown input HOSMO approach. In American Control Conference (ACC), 2013.

[13] U. Nenner, R. Linker, and P. Gutman. Robust feedback stabilization of an unmanned motorcycle. In Robotics, Automation and Mechatronics, 2008.

[14] H.B. Pacejka. Tire and Vehicle Dynamics. SAE International, 2005.

[15] A. Saccon, J. Hauser, and A. Beghi. A virtual rider for motorcycles: An approach based on optimal control and maneuver regulation. In Communications, Control and Signal Processing, 2008.

[16] R. S. Sharp. The stability and control of motorcycles. Mechanical Engineering Science, 13:316 - 329, 1971.

[17] R. S. Sharp and D. J. N. Limebeer. A motorcycle model for stability and control analysis. Multibody System Dynamics, 6:123 - 142, 2001.

[18] R.S. Sharp. Rider control of a motorcycle near to its cornering limits. Vehicle System Dynamics, 50:11931208, 2012.

[19] A. P. Teerhuis and S. T.H. Jansen. Motorcycle state estimation for lateral dynamics. Vehicle System Dynamics, 50(8):1261-1276, 2012.

[20] C. Tseng, B. Chen, and H. Uang. Fuzzy tracking control design for nonlinear dynamic systems via T-S fuzzy model. Fuzzy Systems, IEEE Transactions on, 9(3):381-392, 2001.

[21] S. Yan and Z. Sun. Study on separation principles for ts fuzzy system with switching controller and switching observer. Neurocomputing, 73(13-15):2431 - 2438, 2010.

[22] J. Yi, D. Song, A. Levandowski, and S. Jayasuriya. Trajectory tracking and balance stabilization control of autonomous motorcycles. In Robotics and Automation, 2006. ICRA 2006. Proceedings 2006 IEEE International Conference on, pages 2583-2589, 2006. 